\title{
Observation of periodic variable stars towards the Galactic spiral arms by EROS II $^{\star, \star \star}$
}

F. Derue ${ }^{1,2, \star \star \star}$, J.-B. Marquette ${ }^{3}$, S. Lupone ${ }^{3}$, C. Afonso ${ }^{2,4, \dagger}$, C. Alard $^{5}$, J.-N. Albert ${ }^{1}$, A. Amadon ${ }^{2}$, J. Andersen ${ }^{6}$, R. Ansari ${ }^{1}$, É. Aubourg ${ }^{2}$, P. Bareyre ${ }^{2,4}$, F. Bauer ${ }^{2}$, J.-P. Beaulieu ${ }^{3}$, G. Blanc ${ }^{2, \ddagger}$,

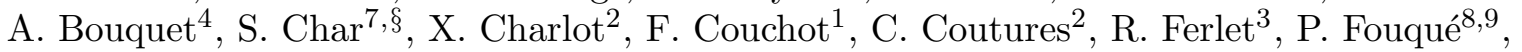
J.-F. Glicenstein ${ }^{2}$, B. Goldman ${ }^{2,4, \dagger}$, A. Gould ${ }^{4,10}$, D. Graff ${ }^{2,11}$, M. Gros ${ }^{2}$, J. Haïssinski ${ }^{1}$, J.-C. Hamilton ${ }^{4}$, D. Hardin ${ }^{2, \|}$, J. de Kat ${ }^{2}$, A. Kim ${ }^{4, \ddagger}$, T. Lasserre ${ }^{2}$, L. Le Guillou ${ }^{2}$, É. Lesquoy ${ }^{2,3}$, C. Loup ${ }^{3}$, C. Magneville ${ }^{2}$,

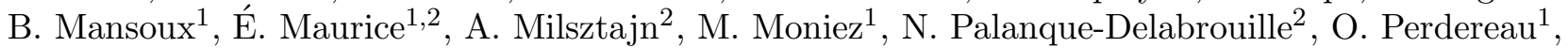
L. Prévot ${ }^{1,2}$, N. Regnault ${ }^{1, \ddagger}$, J. Rich $^{2}$, M. Spiro ${ }^{2}$, A. Vidal-Madjar ${ }^{3}$, L. Vigroux ${ }^{2}$, S. Zylberajch ${ }^{2}$, and The EROS collaboration

${ }^{1}$ Laboratoire de l'Accélérateur Linéaire, IN2P3-CNRS-Université de Paris-Sud, BP 34, 91898 Orsay Cedex, France

2 CEA, DSM, DAPNIA, Centre d'Études de Saclay, 91191 Gif-sur-Yvette Cedex, France

3 Institut d'Astrophysique de Paris, INSU-CNRS, 98bis boulevard Arago, 75014 Paris, France

4 Collège de France, LPCC, IN2P3-CNRS, 11 place Marcelin Berthelot, 75231 Paris Cedex, France

5 DASGAL, INSU-CNRS, 77 avenue de l'Observatoire, 75014 Paris, France

6 Astronomical Observatory, Copenhagen University, Juliane Maries Vej 30, 2100 Copenhagen, Denmark

7 Universidad de la Serena, Facultad de Ciencias, Departamento de Fisica, Casilla 554, La Serena, Chile

8 Observatoire de Paris, DESPA, 92195 Meudon Cedex, France

9 European Southern Observatory (ESO), Casilla 19001, Santiago 19, Chile

10 Ohio State University, Department of Astronomy, Columbus, OH 43210, USA

11 University of Michigan, Department of Astronomy, Ann Arbor, MI48109, USA

12 Observatoire de Marseille, INSU-CNRS, 2 place Le Verrier, 13248 Marseille Cedex 04, France

Received 26 September 2001 / Accepted 11 April 2002

Abstract. We present the results of a massive variability search based on a photometric survey of a six square degree region along the Galactic plane at $\left(l=305^{\circ}, b=-0.8^{\circ}\right)$ and $\left(l=330^{\circ}, b=-2.5^{\circ}\right)$. This survey was performed in the framework of the EROS II (Expérience de Recherche d'Objets Sombres) microlensing program. The variable stars were found among 1913576 stars that were monitored between April and June 1998 in two passbands, with an average of 60 measurements. A new period-search technique is proposed which makes use of a statistical variable that characterizes the overall regularity of the flux versus phase diagram. This method is well suited when the photometric data are unevenly distributed in time, as is our case. 1362 objects whose luminosity varies were selected. Among them we identified 9 Cepheids, 19 RR Lyræ, 34 Miras, 176 eclipsing binaries and 266 Semi-Regular stars. Most of them are newly identified objects. The cross-identification with known catalogues has been performed. The mean distance of the RR Lyræ is estimated to be $\sim 4.9 \pm 0.3 \mathrm{kpc}$ undergoing an average absorption of $\sim 3.4 \pm 0.2$ mag. This distance is in good agreement with that of disc stars that contribute to the microlensing source star population. Our catalogue and light curves are available electronically from the CDS, Strasbourg and from our Web site**.

Key words. techniques: photometry - stars: variables: general - galaxy: stellar content

Send offprint requests to: J.-B. Marquette,

e-mail: marquett@iap.fr

* Full Tables 4 and 5 are only available in electronic form at the CDS via anonymous ftp to

cdsarc.u-strasbg.fr (130.79.128.5) or via

http://cdsweb.u-strasbg.fr/cgi-bin/qcat?J/A+A/389/149

$\star \star$ This work is based on observations made with the MARLY

telescope of the EROS collaboration at the European Southern Observatory, La Silla, Chile.

$\star \star \star$ Presently at Centre de Physique des Particules de Marseille, IN2P3-CNRS, 163 avenue de Luminy, case 907, 13288 Marseille Cedex 09, France.

\footnotetext{
$\dagger$ Presently at Department of Astronomy, New Mexico State University, Las Cruces, NM88003-8001, USA.

$¥$ Presently at University of California, Department of Physics, Berkeley, CA 97720, USA.

$\S$ Deceased.

ๆ Presently at ISN, IN2P3-CNRS-Université JosephFourier, 53 avenue des Martyrs, 38026 Grenoble Cedex, France.

\| Presently at LPNHE, IN2P3-CNRS-Universités Paris VI et VII, 4 place Jussieu, 75252 Paris Cedex 05, France.

** http://eros.in2p3.fr
} 


\section{Introduction}

In 1996, the EROS II collaboration started an observation program towards the Galactic Spiral Arms (GSA) dedicated to microlensing events. Since then, four regions of the Galactic plane located at large angles with respect to the Galactic Centre are being monitored to disentangle the disc, bar and halo contributions to the microlensing optical depth. Seven microlensing event candidates have already been published, based on three years (1996-98) of observations (Derue et al. 1999, 2001), (hereafter Papers I and II). The distance of the source stars used in these papers to compute the expected optical depths was deduced from a detailed study of our colour-magnitude diagrams. It was thus found that the source star population is located $\sim 7 \mathrm{kpc}$ away, undergoing an interstellar extinction $A(V)$ of about 3 mag (see Mansoux 1997 for more details). This distance estimate is in rough agreement with the distance to the spiral arms obtained by Georgelin et al. (1994) and Russeil et al. (1998), but its uncertainty is limiting further interpretation of our microlensing optical depth estimates. It was therefore desirable to seek more information on the distance distribution of the source stars - whether these stars belong to the disc or to the spiral arms - and on the reddening along our observation line of sights. This led us to perform a dedicated variable star search between April and June 1998, on a subset of our Galactic plane fields. The analysis was restricted to the brightest stars of this subset.

Among the wide variety of variable stars, periodic ones are of particular interest. The properties of Cepheids make them well suited to trace the Galactic spiral arms. Their reddening is measurable as well as their distance via the period-luminosity (PL) relation. RR Lyræ stars are old stars, well suited to trace the disc population. One can infer their mean dereddened magnitude and their absolute magnitude (Gould \& Popowski 1998). The infrared $\mathrm{PL}(\mathrm{K})$ relation for Miras and Semi-Regular variable stars can be calibrated using a comparison of DENIS and EROS LMC giant stars (Cioni et al. 2001). Finally detached eclipsing binaries also offer the opportunity to measure their stellar parameters and their distance (Paczyński 1996).

This paper presents the results of this particular campaign that led to a catalogue containing a large number of new variable objects in the Galactic plane. Section 2 gives the basic features of the observational setup, Sect. 3 gives details on a new algorithm used to search for periodic variations of the luminosity. Section 4 describes the catalogue and the cross-identification process. In Sect. 5 we use the selected RR Lyræ to estimate the mean reddening of our fields and we give the distance distribution of these stars.

\section{Experimental setup and observations}

The MARLY telescope and its two cameras, the way we carry out our observations, as well as our data reduction sequence are described in Paper I and references therein.
Table 1. Characteristics of the 6 one square degree fields monitored for this study. The equatorial (J2000) and galactic coordinates of the field centres, the number of photometric measurements per light curve per colour $N_{\mathrm{m}}$ and the number of analysed light curves $N_{\text {obs }}$ (in millions) are given for each field. The observation duration was $\sim 100$ days.

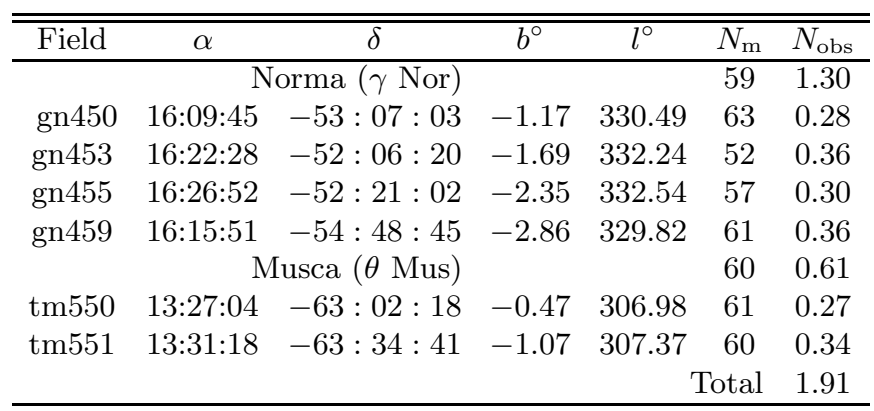

The two EROS passbands are non standard. The socalled EROS-red passband $R_{\mathrm{E}}$ is centred on $\bar{\lambda}=762 \mathrm{~nm}$, close to $I_{\mathrm{C}}$ Cousins, with a full width at half maximum $\Delta \lambda \simeq 85 \mathrm{~nm}$, and EROS-visible passband $V_{\mathrm{E}}$ is centred on $\bar{\lambda}=600 \mathrm{~nm}$, close to $V_{\mathrm{J}}$ Johnson, with $\Delta \lambda \simeq 78 \mathrm{~nm}$. The EROS II colour magnitude system is defined as follows: a zero colour star with $V_{\mathrm{E}}-R_{\mathrm{E}}=0$ (a main sequence A0 star) will have its $R_{\mathrm{E}}$ magnitude numerically equal to its Cousins $R_{\mathrm{C}}$ magnitude and its $V_{\mathrm{E}}$ magnitude numerically equal to its Johnson $V_{\mathrm{J}}$ magnitude. The colour transformation between the EROS II system $\left(V_{\mathrm{E}}, R_{\mathrm{E}}\right)$ and the standard Johnson-Cousins $\left(V_{\mathrm{J}}, I_{\mathrm{C}}\right)$ system is then:

$I_{\mathrm{C}}=R_{\mathrm{E}}+0.01 \times\left(V_{\mathrm{E}}-R_{\mathrm{E}}\right)$

$V_{\mathrm{J}}=V_{\mathrm{E}}+0.39 \times\left(V_{\mathrm{E}}-R_{\mathrm{E}}\right)$.

The colour coefficients are obtained from the study of our passbands based on Landolt standards and on one of the EROS II LMC fields observed simultaneously in $B V_{\mathrm{J}} R_{\mathrm{C}} I_{\mathrm{C}}$ with the Danish $1.54 \mathrm{~m}$ (at ESO-La Silla) and with the MARLY. The zero points are established with tertiary standards in $B V R I$ taken with the Danish $1.54 \mathrm{~m}$ (Regnault 2000). We have cross-checked our $R_{\mathrm{E}}$ photometry with the $I_{\mathrm{C}}$ one of DENIS (Fouqué et al. 2000). Furthermore, using Eqs. (1), the mean magnitudes of the LMC red-giant clump stars agree within $0.1^{\text {mag }}$ with determinations made by Harris \& Zaritsky (1999) and Udalski et al. (1998). We thus estimate that the precision of the zero points of the MARLY calibration is $\sim 0.1^{\text {mag }}$.

Among the 29 fields of the EROS GSA microlensing program, the six fields considered here were monitored about once per night between April and June 1998. They represent 2 square degrees towards $\theta$ Mus and 4 towards $\gamma$ Nor (named after the closest bright star). Table 1 gives their coordinates, the number of images $N_{\mathrm{m}}$ taken in each direction and the number of analysed light curves $N_{\text {obs }}$. To avoid CCD saturation by the brightest stars $\left(I_{\mathrm{C}} \sim 9\right)$, by Cepheids or Miras in particular, we have reduced the exposure time to $15 \mathrm{~s}$ instead of the $120 \mathrm{~s}$ used in the microlensing survey. As a consequence the catalogue is incomplete as far as faint stars are concerned, but it could be 


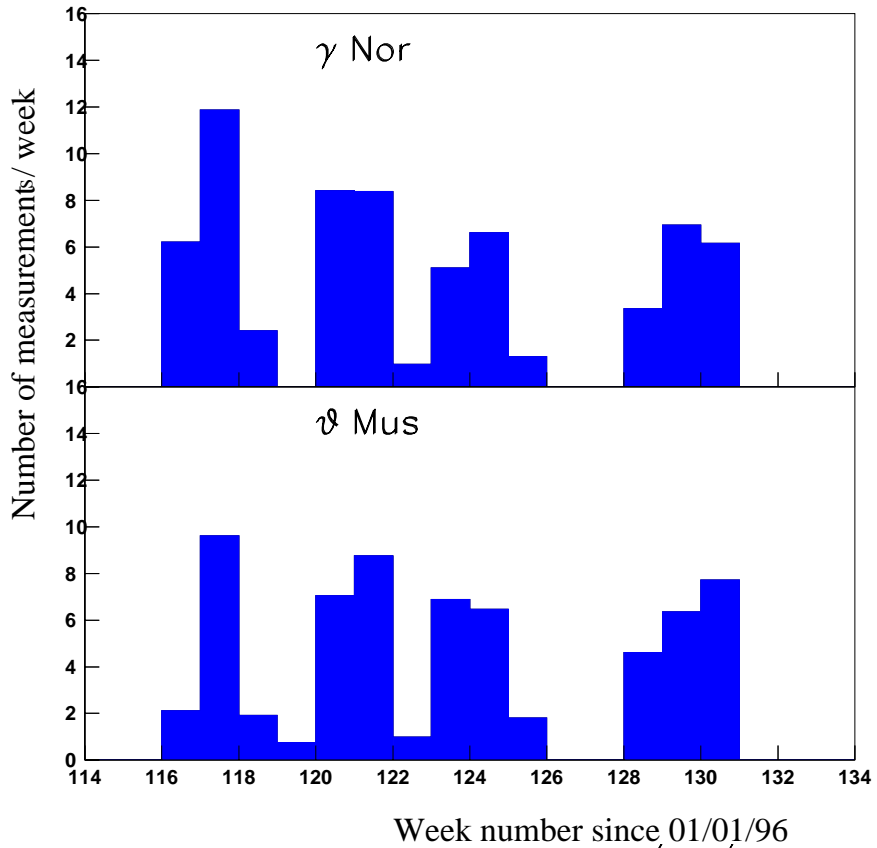

Fig. 1. Average time sampling for the 6 fields monitored ( 2 towards $\gamma$ Nor, upper panel, and 4 towards $\theta$ Mus, lower panel), in number of measurements per week and per field.

updated later by using the total set of available GSA images. Figure 1 shows the average time sampling. Three gaps can be seen in our data: the first two (around weeks 119 and 123) were due to bad weather conditions while the third one (around week 127) corresponds to the annual maintenance of our setup.

\section{Search for periodic stars}

\subsection{Reconstruction of the light curves}

Since the EROS photometry is described in detail in Ansari (1996) only the main features of the PEIDA++ package are summarised below. For each field, a template image is first constructed using one exposure of very good quality. A reference star catalogue is set up with this template using the CORRFIND star finding algorithm (Palanque-Delabrouille et al. 1998). For each subsequent image, after geometrical alignment with the template, each identified star is fitted together with its neighbours, using a PSF determined on bright isolated stars and imposing the position from the reference catalogue. A relative photometric alignment is then performed, assuming that most stars do not vary. Photometric errors are computed for each measurement, assuming again that most stars are stable, and parameterised as a function of star brightness and image sequence number. Figure 2 shows the mean point-to-point relative dispersion of the measured fluxes along the light curves as a function of $R_{\mathrm{E}}$ and $V_{\mathrm{E}}$. The photometric accuracy is $\sim 15 \%$ at $R_{\mathrm{E}} \sim 18$, and about $2 \%$ for the brightest stars. Finally, using the PEIDA + + photometric package, we reconstruct the light curves of 1913576 stars.
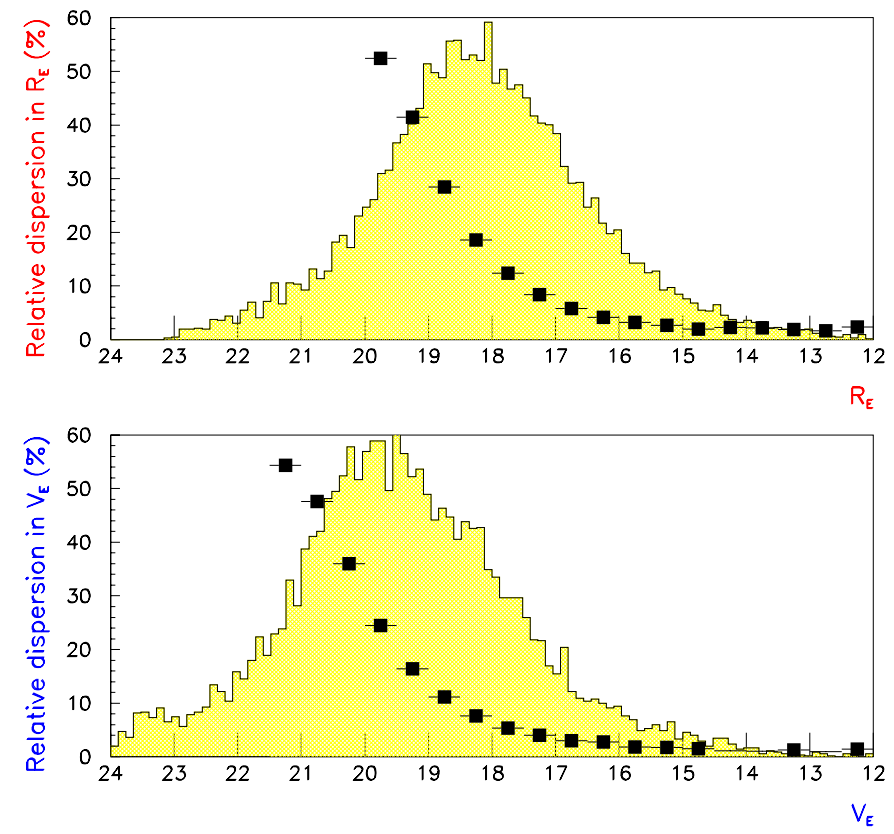

Fig. 2. Average value of the relative frame to frame dispersion of the luminosity measurements versus $R_{\mathrm{E}}$ (upper panel) and $V_{\mathrm{E}}$ (lower panel), for stars with at least 30 reliable measurements in each colour. This dispersion is taken as an estimator of the mean photometric precision. The superimposed hatched histograms show the EROS magnitude distribution of monitored stars.

Table 2. Impact of each selection criterion on the data. For each cut, the number of remaining light curves is given.

\begin{tabular}{llr}
\hline \hline Cut & Criterion & \\
\hline & Total analysed & 1913576 \\
1 & $N_{\mathrm{m}}>30$ & 1299690 \\
2 & $R_{\mathrm{E}}<17$ & 330089 \\
3 & Pre-filtering & 41545 \\
4 & Period search & 2553 \\
5 & Aliasing & 2424 \\
6 & Visual inspection & 1362 \\
\hline
\end{tabular}

\subsection{Pre-selection}

Each one of the light curves is subjected to a series of selection criteria in order to isolate a small sub-sample on which we will apply the time consuming period search algorithms. These analysis cuts are briefly described hereafter (see Derue 1999 for more details) and their effect on the data is summarised in Table 2:

cut 1: At least 30 measurements should be available in both passbands and the base flux must be positive;

cut 2: The search is restricted to stars whose magnitude is $R_{\mathrm{E}}<17$ which corresponds to a photometric accuracy in $R_{\mathrm{E}}$ better than $\sim 10 \%$.

cut 3: A non specific pre-filter is applied which retains most variable stars. It selects light curves satisfying one or both of the following criteria:

- the relative dispersion of the flux measurements is $25 \%$ larger than the average one for the set of stars having the same magnitude; 
- the distribution of the deviations with respect to the base flux is incompatible with the one expected from a stable source with Gaussian errors during the observation period (Kolmogorov-Smirnov test).

These cuts are tuned to select $\sim 10 \%$ of the light curves. We have checked that this procedure allows one to retrieve the previously known Cepheids observed by EROS in the Magellanic Clouds. We also keep a randomly selected set of light curves $(\sim 2 \%)$ to produce unbiased colour-magnitude diagrams, for comparison purposes;

At this stage a set of 41545 light curves remains which is then subjected to a periodicity search.

\subsection{Light curve selection}

We use three independent methods to extract periodic light curves. The first two are classical methods already described in the literature: method 1 is based on the Lomb-Scargle periodogram (see Scargle 1982) while method 2 makes use of the One Way Analysis of Variance algorithm (see Schwarzenberg-Czerny 1996). Both provide the probability for false periodicity detection. In method 1 one computes the Fourier power over a set of frequencies. It is therefore well adapted to identify sinusoidal light curves. It can be improved by incorporating higher harmonics in order to detect any kind of variability such as eclipsing binaries (Grison 1994; Grison et al. 1995).

We developed a new method, the third one, in order to extract periodic light curves in a way which is insensitive to the particular shape of the variation. This method also provides a probability for false periodicity detection.

It consists in searching for a frequency such that the corresponding phase diagram, i.e. the series of fluxes $F_{i}$ versus phases $\varphi_{\mathrm{i}}$ in increasing order of $\varphi$, displays a regular structure significantly less scattered than for other frequencies. Let $T_{\text {obs }}$ be the observation duration $(\sim 100$ days in this analysis). We span the frequency domain from $T_{\mathrm{obs}}^{-1}$ to $(0.2 \text { days })^{-1}$ with a constant step of $\left(4 \times T_{\text {obs }}\right)^{-1}$. The total number of test-frequencies is thus $N_{\text {test }} \sim 2000$. This sampling ensures that the total phase increment over $T_{\text {obs }}$ is $\pi / 2$ for two adjacent test-frequencies.

For each value of the test-frequency we compute the corresponding phase diagram. We calculate a $\chi^{2}$ from the weighted differences of $F_{i}$ and the fluxes interpolated between $F_{i-1}$ and $F_{i+1}$ :

$\chi^{2}=\sum_{i=1}^{N_{m}}\left(\frac{F_{i}-\left(1-R_{i}\right) \times F_{i-1}-R_{i} \times F_{i+1}}{\sigma_{i}}\right)^{2}$

where $R_{i}=\left(\varphi_{i}-\varphi_{i-1}\right) /\left(\varphi_{i+1}-\varphi_{i-1}\right)$ and $N_{\mathrm{m}}$ is the number of measurements. The uncertainty $\sigma_{i}$ takes into account the errors $\epsilon_{i}$ on the flux $F_{i}$ and on the interpolated flux: $\sigma_{i}^{2}=\epsilon_{i}^{2}+\left(1-R_{i}\right)^{2} \epsilon_{i-1}^{2}+R_{i}^{2} \epsilon_{i+1}^{2}$. Expression (2) can be interpreted as the $\chi^{2}$ of the set of differences between the odd measurements with respect to the line joining even
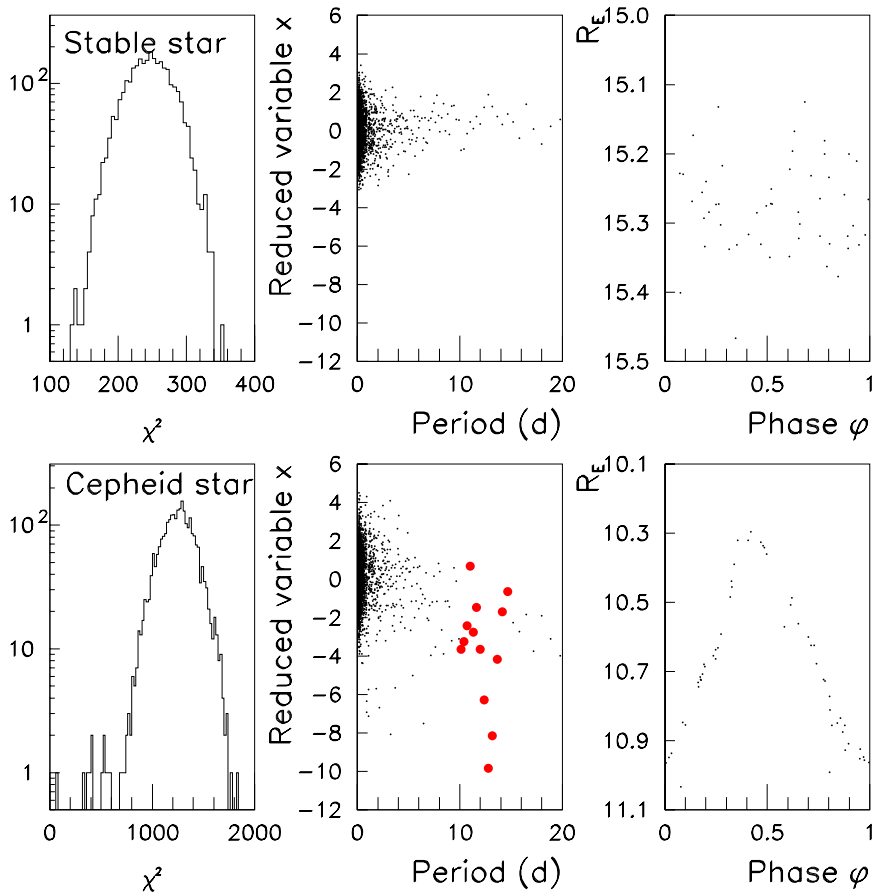

Fig. 3. Distributions of $\chi^{2}$ (left panels) and variation of $x$ with the value of the test-period in days (middle panels) obtained with the third method for a stable star and for a typical Cepheid candidate. The bold dots are pointing to the actual period of the star. The right panels show the light curve (in $R_{\mathrm{E}}$ ) obtained once the period has been folded in.

ones, added to the $\chi^{2}$ of the set of differences between even measurements with respect to the line joining odd ones. If the star is measured in both colours, we add the $\chi^{2}$ obtained in each colour ( $N_{\mathrm{m}}$ is then twice as large). For a given stable star with Gaussian errors, each phase diagram can be considered as a random realisation of the light curve. When the test-frequency spans the search domain, the distribution of the $\chi^{2}$ parameter defined by Eq. (2) is the one of the standard $\chi^{2}$ with $N_{\mathrm{m}}$ degrees of freedom. Since $N_{\mathrm{m}}$ is large enough, this distribution is close to a Gaussian with average $N_{\mathrm{m}}$ and variance $2 N_{\mathrm{m}}$ (see upper left panel of Fig. 3). For a periodic variable star, the $\chi^{2}$ distribution displays a main cluster, when the test-frequency results in a phase diagram with non-correlated point to point variations, and a few lower values when the testfrequency corresponds to a phase diagram with a regular structure (see lower left and middle panels of Fig. 3). In practice, instead of using the parameter defined by Eq. (2), we use the reduced variable:

$x=\frac{\chi^{2}-<\chi^{2}>}{<\chi^{2}>/ N_{\mathrm{m}}} \times \frac{1}{\sqrt{2 N_{\mathrm{m}}}}$

where $\left\langle\chi^{2}>\right.$ is the average of the realisations of $\chi^{2}$ for all test-frequencies. For a stable star, the distribution of this variable $x$ is a Gaussian centred at zero, with unit variance. If the errors are correctly determined $\left\langle\chi^{2}\right\rangle / N_{\mathrm{m}}$ is close to unity; if the errors are all systematically overestimated (or underestimated), then including this term ensures a global renormalisation of the 

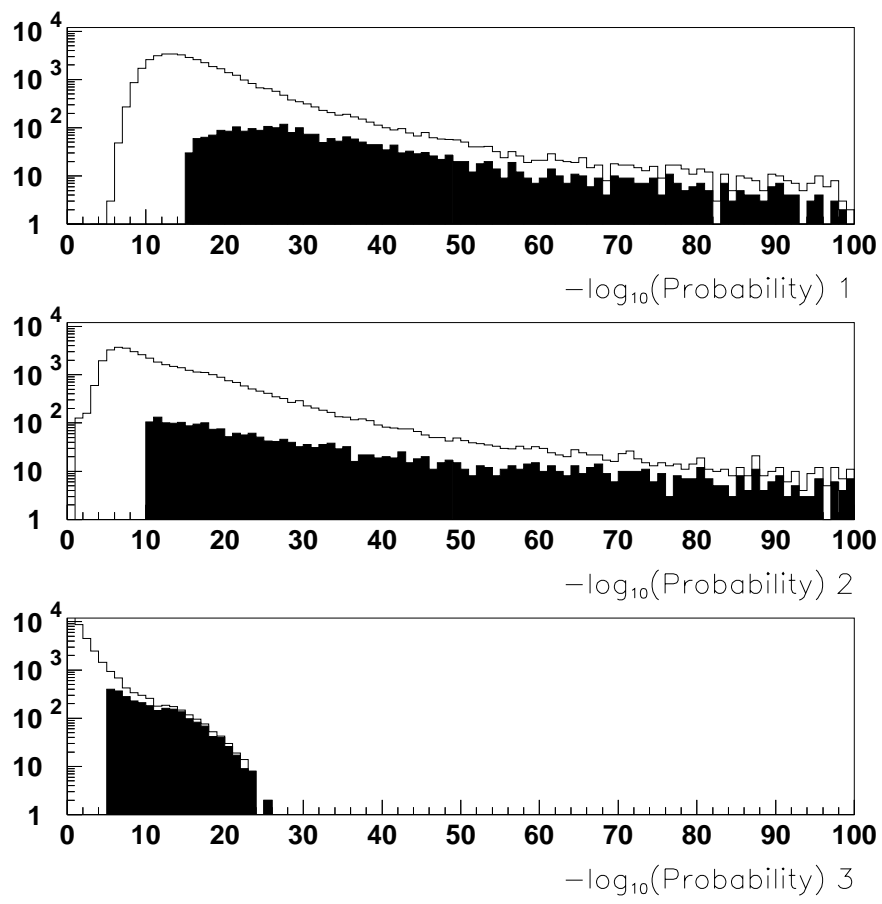

Fig. 4. Distribution of $-\log$ (Prob) for method 1 (top), 2 (middle) and 3 (bottom). The histograms show the distribution for all 41545 stars on which the periodicity search is done (in white) and for the 2553 selected light curves (in black).

errors in Eq. (3), and the distribution of our reduced variable $x$ is also a normal distribution. Let $x_{\min }$ be the smallest value of $x$ calculated among all test-frequencies for a given star. Under the hypothesis that the light curve is produced by a stable star, the probability to obtain at least one value $x \leq x_{\min }$ in a series of $N_{\text {test }}$ realisations is $\operatorname{Prob}\left(x<x_{\min } \mid N_{\text {test }}\right)=1-\left[1-\frac{1}{\sqrt{2 \pi}} \int_{-\infty}^{x_{\min }} e^{-x^{2} / 2} \mathrm{~d} x\right]^{N_{\text {test }}}$. If this probability is small, then:

$\operatorname{Prob}\left(x<x_{\text {min }} \mid N_{\text {test }}\right) \simeq \frac{N_{\text {test }}}{2} \operatorname{erfc}\left(\frac{-x_{\text {min }}}{\sqrt{2}}\right)$.

If the light curve exhibits periodic variations, then there exist test-frequencies for which $x$ is significantly smaller than typical values of this variable (see middle and right panels of Fig. 3), and the probability for false detection is then extremely small. Figure 4 displays the probability distribution obtained with the three methods for the set of filtered light curves ${ }^{1}$.

We apply the three algorithms which all give a probability for no periodicity. A star is accepted only if selected by all three methods with the following thresholds: $P(\operatorname{method} 1)<10^{-15}, P(\operatorname{method} 2)<10^{-10}$, $P(\operatorname{method} 3)<10^{-5}$, tuned in order to allow one to retrieve the previously known Cepheids observed by EROS in the Magellanic Clouds (see Derue 1999 for more details). This procedure (cut 4) selects a sample of 2553 stars.

1 The third method gives a probability which is always larger than $10^{-30}$. Indeed, the smallest value of $x$ which can be obtained takes place when $\chi^{2}=0$, then $|x| \sim \sqrt{N_{\mathrm{m}}}<10$.
Ten times more stars would have been selected if we had used method 1 or method 2 only (with the same thresholds), most of them being spurious variables. The third method would have added far less candidates if used alone, but still a factor 2 more. Combining independent methods has thus the advantage of considerably reducing the noise background (mostly due to aliases of one day or noisy measurements) while giving redundant information about the period. To obtain individual periods we perform Fourier fits with five harmonics:

flux $=$ flux $_{0}+\sum_{l=1}^{5} a_{l} \cos \left(\frac{2 \pi}{P} l\left(t-t_{0}\right)+\phi_{l}\right)$,

where $P$ is the period, $\phi_{l}$ the phase and $a_{l}$ the amplitude. We define the amplitude ratios $R_{k l}=a_{k} / a_{l}$, and the phase differences (defined modulo $2 \pi$ ) $\Phi_{k l}=\phi_{k}-k \phi_{l}$, with $k>l$. Objects with non-significant harmonic amplitudes (i.e. with almost sinusoidal light curves) have $R_{21} \sim 0$ and their $\Phi_{21}$ is ill defined.

The selection of periodic variable stars is complicated by aliases. Some of the stars with periods equal to a simple fraction or a low multiple of one day may be badly phased because of the nightly sequence of measurements. These aliased periods are seen in Fig. 5 as vertical groups of dots at $2 / 3,2$ and 3 days. To eliminate them we demand (cut 5 ) that the fitted periods are not within $\pm 1 \%$ of these values. One can also notice some vertical groups of points around 25 days which correspond to data gaps in our sample (see Fig. 1). Once these objects are removed, 2424 stars remain. The flux values of the remaining stars are folded using each period obtained with the three methods. The resulting phase diagrams are visually inspected. Some of them display an obvious spurious periodic or quasi-periodic variability due to a low photometric quality. After this final visual selection (cut 6) the list of variable stars includes 1362 candidates which exhibit unambiguous periodic variability.

\subsection{Type of variability}

The classification of the selected stars among different types of variability cannot be based on the position of the objects in the colour-magnitude diagram since the spread in distance of these stars entails a spread in magnitude and colour. It is desirable however to classify the various light curves according to some physical parameters. In the following we mainly use criteria based on the period $P$ of the luminosity variations and on the amplitude ratio $\Delta V_{\mathrm{E}} / \Delta R_{\mathrm{E}}$. For each selected type of variable star the phase diagram of a typical candidate is displayed in Fig. 6. Three groups are distinguished depending on their period:

1st: stars with a period larger than 60 days (543 objects): For these objects an entire period has not been observed. There is thus no warranty that these objects are periodic ones. 34 display a nearly linear light curve and are thus catalogued as Miras candidates. 


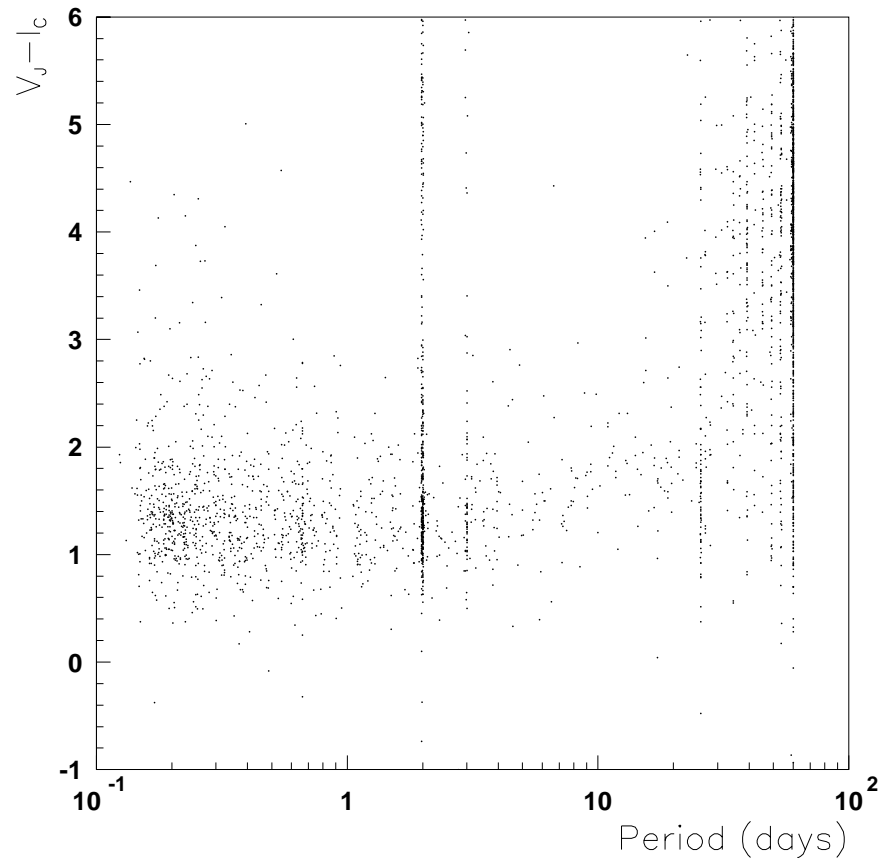

Fig. 5. Period-colour diagram $\left(P\right.$ in days vs. $\left.V_{\mathrm{J}}-I_{\mathrm{C}}\right)$ of the 2553 selected candidates before cut 5 .

The other 509 objects are catalogued as Long Period Variable stars (LPVs).

2nd: stars with a period between 30 and 60 days (387 objects):

264 Semi-Regular variable stars are selected by requiring: $\Delta V_{\mathrm{E}} / \Delta R_{\mathrm{E}}>1.2$ to select pulsating stars (see below), $V_{\mathrm{J}}-I_{\mathrm{C}}>2.5$ to discriminate from bluer variable stars and $\Delta R_{\mathrm{E}}<1.0$ to avoid possible Miras or LPVs wrongly phased. The long term stability of these stars is not known. Some of the reported periods may change from season to season, as a result of their semi-regular behaviour. The remaining 123 objects are catalogued as miscellaneous variable stars.

3rd: stars with a period smaller than 30 days (432 objects):

The colour change for a Cepheid in standard passbands is $\Delta V_{\mathrm{J}} / \Delta I_{\mathrm{C}}>1.3$ (Madore et al. 1991) which corresponds to $\Delta V_{\mathrm{E}} / \Delta R_{\mathrm{E}}>1.2$ in the EROS system. Two sets are thus distinguished based on this criterion:

- The pulsating variable stars (60 objects):

For stars with period $P<1$ day, two samples of RR Lyræ are identified: the RRc have $R_{21}<$ 0.4 (14 objects) and the RRab have $R_{21}>0.4$ (5 objects). We adopt the morphological classification proposed by Antonello et al. (1986) and classify as $s$-Cepheids the stars that lie in the lower part of the $R_{21}-P$ plane, and as classical Cepheids the remaining stars. $s$-Cepheids pulsate in the first overtone and classical Cepheids in the fundamental mode (see e.g. Beaulieu et al. 1995; Beaulieu \& Sasselov 1996). We use the
Table 3. Number of selected objects for each type of variability.

\begin{tabular}{lll}
\hline \hline Period range & Type & Number of objects \\
\hline$P>60 \mathrm{~d}$ & & 543 \\
& LPV & 509 \\
& Miras & 34 \\
& & 387 \\
$P<30 \mathrm{~d}$ & Semi-Regular & 264 \\
& miscellaneous & 123 \\
& pulsating & 432 \\
& RRc & 60 \\
& RRab & 14 \\
& classical-Cepheids & 6 \\
& $s$-Cepheids & 3 \\
& miscellaneous & 32 \\
& non-pulsating & 372 \\
& EA & 130 \\
& EB & 35 \\
& EW & 11 \\
& miscellaneous & 196 \\
\hline
\end{tabular}

empirical function $R_{21}^{\text {cut }}(P)=0.4-(P / 30$ days $)$ in order to separate these pulsation modes (see Fig. 7). Among the five objects that pass the $s$ Cepheid cut, only three belong to the $R_{21}-P$ and $\Phi_{21}-P$ distributions of galactic $s$-Cepheids and their phase parameter $\Phi_{21}$ is poorly constrained. Besides most of classical Cepheids have amplitudes larger than $\Delta R_{\mathrm{E}}>0.4^{\mathrm{mag}}$ (see e.g. Afonso et al. (1999)). For stars with period $P>1$ day, three samples are then identified: the classical Cepheids have $R_{21}>R_{21}^{\text {cut }}$ and $\Delta R_{\mathrm{E}}>0.4^{\mathrm{mag}}$ (6 objects); the $s$-Cepheids have $R_{21}<R_{21}^{\text {cut }}$ and $3 \mathrm{rad}<\Phi_{21}<6 \mathrm{rad}$ (3 objects); the remaining 32 objects are catalogued as miscellaneous pulsating stars.

- The non-pulsating variable stars (372 objects): The remaining objects have similar amplitudes in both passbands. We classify them according to the following criteria. Algol systems (130 stars, type EA) display well-defined eclipses whose secondary one has a depth lower than half the primary one, and possibly flat light curve between them. EB type objects (35 in total) show a secondary eclipse equal to half the primary one. The EW type (11 objects) is characterised by similar depths of the two eclipses. The members of a residual sample of 196 objects do not look like convincing eclipsing binaries and are catalogued as miscellaneous variable stars.

As emphasised by Udalski et al. (1999a) a large number of variable objects show small amplitude sinusoidal variations, such as ellipsoidal binary variable stars. A contamination of the sample of pulsating stars by eclipsing binaries is thus possible. 

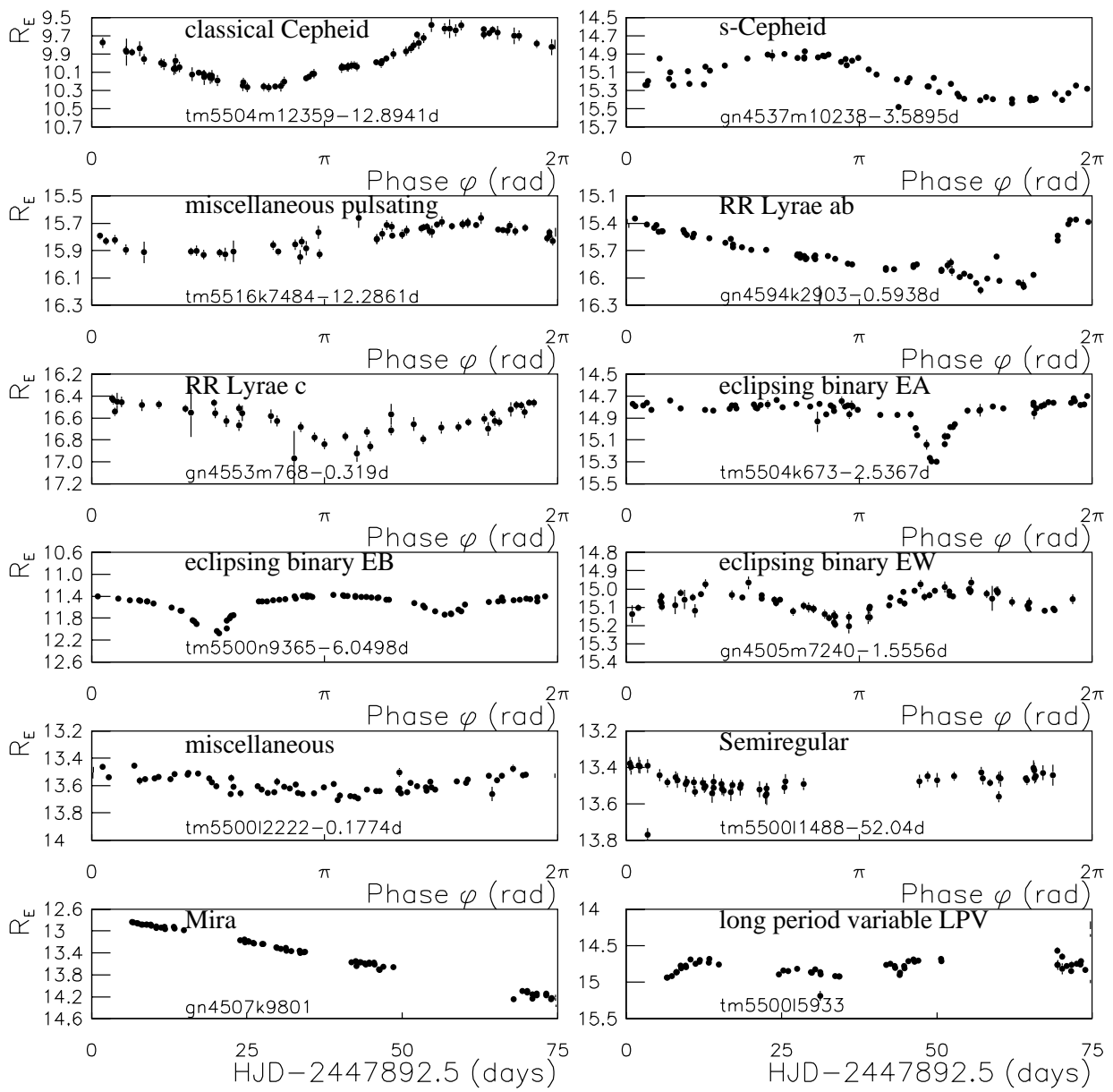

Fig. 6. Phase diagrams (or light curves for the two lowest panels) for typical variable stars of our catalogue in $R_{\mathrm{E}}$ magnitude.

Figures 8 and 9 show the location of the selected variables in the colour-magnitude diagrams. Also plotted are 10000 stars located in the central part of the two fields tm550 and gn450. Most of the Cepheids are much brighter than our magnitude threshold (cut 2); this is not so for RR Lyræ (see Fig. 9, lower panel). Our catalogue is thus not complete for this type of variable stars, as already mentionned.

\section{The catalogue}

The catalogue is composed of two tables containing objects with periods $P$ smaller or larger than 30 days, respectively. The identifier of each star is given according to the recommendations of the IAU Commission 5 in The Rules and Regulations for Nomenclature (see the Annual Index of A\&A). The general acronym used in the catalogue is EROS2 GSA followed by J2000 equatorial coordinates in the format JHHMMSS \pm DDMMSS. The remainder of the identifier in parentheses gives some information relating to the internal organisation of the EROS database: gnnnn or tmnnn is the name of the field, followed by the CCD number and the location on the image following the EROS II nomenclature. The remaining number is the star identifier used in the EROS database. As an example, J132630-630945(tm5504m12359) is the name of the 12359th star observed in quarter $\mathrm{m}$ of CCD 4 in the field tm550. The J2000 equatorial coordinates of this star are 13:26:30.11, $-63: 09: 45.66$.

The equatorial coordinates (J2000) of individual stars have been obtained as follows. First, we have inserted the suitable WCS keywords into the header of the EROS II reference images using the WCSTOOLS package (Mink 1999). Whenever possible, the cross-identification of each star with previously known objects within a $10^{\prime \prime}$ search radius has been done using the SIMBAD and VIzIER databases available at the CDS, Strasbourg.

The tables contain the following information:

1. Identifier;

2. Right ascension $\alpha$ (J2000);

3. Declination $\delta(\mathrm{J} 2000)$;

4. $\left\langle R_{\mathrm{E}}\right\rangle$ mean magnitude in EROS-red;

5. $\Delta R_{\mathrm{E}}$ amplitude peak to peak in $R_{\mathrm{E}}$;

6. $\left\langle V_{\mathrm{E}}\right\rangle$ mean magnitude in EROS-visible;

7. $\Delta V_{\mathrm{E}}$ amplitude peak to peak in $V_{\mathrm{E}}$;

8. Period in days. Note that periods longer than 30 days are given with less accuracy since the time span of the measurements does not allow a precise determination. Measured periods which are longer than 60 days (i.e. $2 / 3$ of the observation period) are flagged by writing " $P>60 \mathrm{~d}$ " and have no warranty to be true periodic 

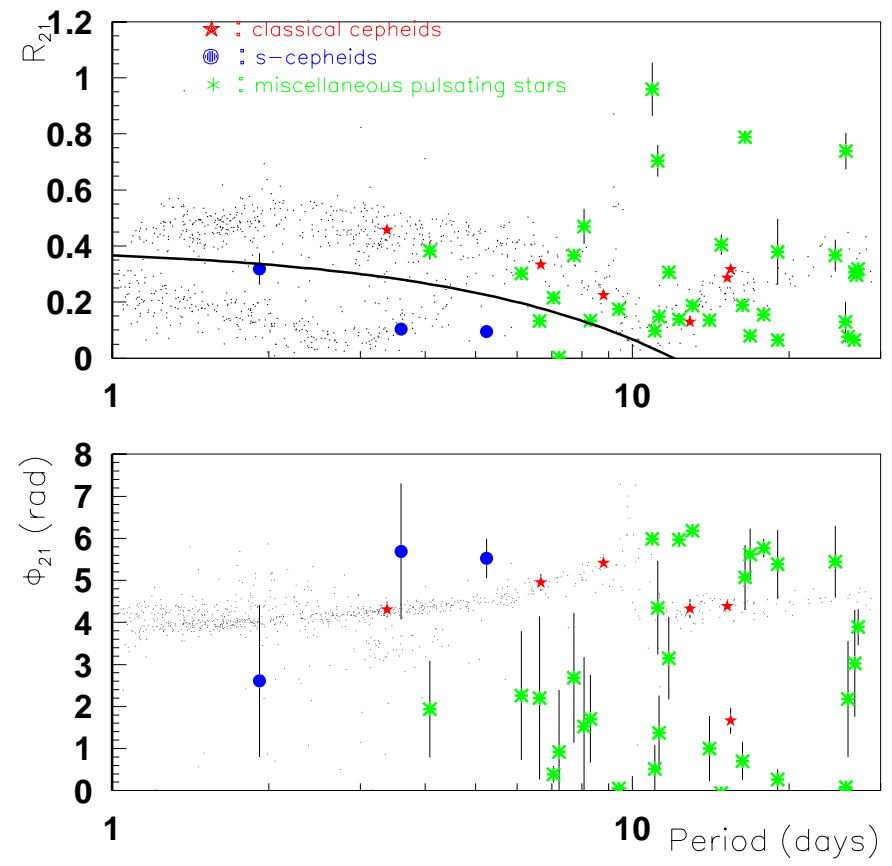

Fig. 7. Distinction between classical Cepheids, $s$-Cepheids and miscellaneous pulsating stars in the $R_{21}-P$ and $\Phi_{21}-P$ planes. The individual uncertainties are reported. The cloud of points represent the Cepheids observed in the LMC (adapted from Afonso et al. 1999). The curve corresponds to the empirical function $R_{21}^{\text {cut }}(P)=0.4-(P / 30$ days $)$ used to distinguish between $s$ and classical Cepheids.

variable stars. The peak to peak amplitude of these stars could be meaningless and the mean magnitude is determined with low accuracy.

For Cepheids and RR Lyræ the results of the Fourier fit are given:

9. Fourier coefficient ratio $R_{21}$;

10. Fourier coefficient ratio $R_{31}$;

11. Phase difference $\phi_{21}$ (in rad);

Also given when possible:

13. Type of variability $(\mathrm{C}=$ classical Cepheids, $\mathrm{S}=s$ Cepheids, puls. $=$ miscellaneous pulsating stars, EA, EB, EW = eclipsing binaries, misc $=$ miscellaneous variable stars, $\mathrm{SR}=$ Semi-Regular variables, $\mathrm{M}=$ Miras, $\mathrm{LPV}=$ long period variables);

14. Name of cross-identified object(s) within a search radius of $10^{\prime \prime}$.

Tables 4 and 5 show a sample of the catalogue that we plan to install at the CDS (see also our Web site http://eros.in2p3.fr/).

A by-product of such a survey is the possibility to update the coordinates given in older catalogues. As an example, it was found that the well known classical Cepheid OO Cen with the SIMBAD identifier $\mathrm{V}^{*} \mathrm{OO}$ Cen was $20^{\prime \prime}$ away from an EROS object, namely J132630630945(tm5504m12359), as shown in the finding chart of Fig. 10. The period and coordinates we report are compatible with the ones given by the General Catalogue
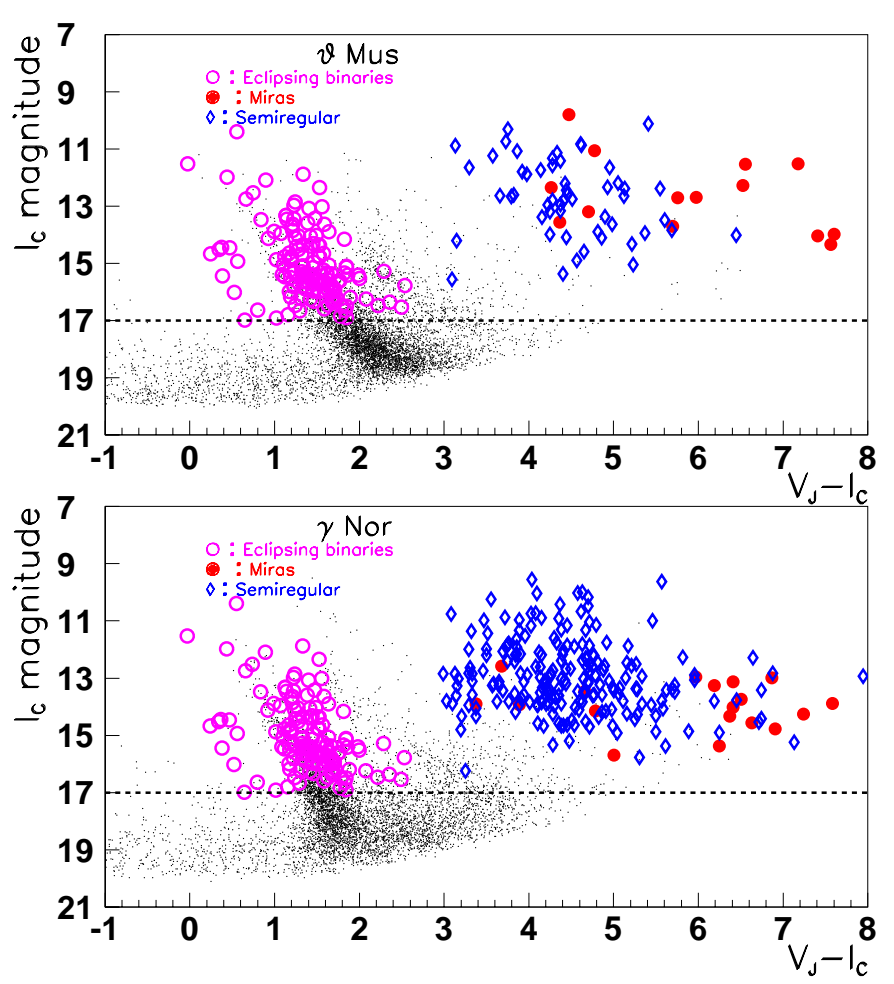

Fig. 8. Colour-magnitude diagrams $\left(I_{\mathrm{C}}\right.$ vs. $\left.V_{\mathrm{J}}-I_{\mathrm{C}}\right)$ for the Miras (represented by dots $\bullet$ ), Semi-Regular variable stars (diamonds $\diamond$ ) and eclipsing binaries (open circles $\circ$ ). The dotted line corresponds to cut 2 on the luminosity of the stars.

of VARIable Stars (GCVS) ${ }^{2}$ (Kholopov 1985) (see Table 6). It seems that an error occurred when the coordinates of this particular star were filled in the SIMBAD. The light curve of this object is shown in Fig. 6 .

We have performed several cross-identifications of our catalogue with those previously available, namely the IRAS Point Source Catalog (Beichman et al. 1998), the MSX5C Infrared Astrometric Catalog (Egan et al. 1996), the Two Micron All Sky Survey (2MASS) (Skrutskie et al. 1997), the CKS91 catalogue (Caldwell et al. 1991) and the General Catalogue of Variable Stars (GCVS) (Kholopov 1985). A total of 38 IRAS sources and 220 MSX5C objects have been thus retrieved. The overlap with the available 2MASS catalogue exists only for the EROS field gn459, representing 255 stars. A total of 2332 MASS objects have been thus retrieved among them 37 objects classified as SemiRegular variables.

An overlap exists with the CKS91 catalogue (Caldwell et al. 1991). These authors have searched for bright Cepheids and other variable stars with $I<14$, towards Crux and Centaurus, during 42 days with less than 10 measurements per star. A small overlap exists between this survey and the GSA fields tm550 and tm551. Unfortunately this overlap involves our CCD \#2 which was not operational at the time of the

\footnotetext{
${ }^{2}$ Queries on this catalogue are possible at http://www.sai.msu.su/cgi-bin/wdb-p95/gcvs/stars/form
} 
Table 4. Catalogue of stars with $P<30$ days (sample).

\begin{tabular}{|c|c|c|c|c|c|c|c|c|c|c|c|c|c|c|c|}
\hline EROS2 GSA ID & $\mathrm{RA}(\mathrm{J} 2000)$ & $\mathrm{DEC}(\mathrm{J} 2000)$ & $\left\langle R_{\mathrm{E}}\right\rangle$ & $\Delta R_{\mathrm{E}}$ & $\left\langle V_{\mathrm{E}}\right\rangle$ & $\Delta V_{\mathrm{E}}$ & $P($ days $)$ & $R_{21}$ & $\sigma_{R_{21}}$ & $R_{31}$ & $\sigma_{R_{31}}$ & $\phi_{21}$ & $\sigma_{\phi_{21}}$ & Type & Note \\
\hline $\mathrm{J} 132357-631158(\mathrm{tm}$ & 13:23:57.99 & $-63: 11: 58.88$ & 14.73 & 0.58 & 15.7 & 0.64 & 2.5367 & & & & & & & EA & \\
\hline J132402-633439(tm5506k1244) & 13:24:02.90 & $-63: 34: 39.66$ & 4.20 & 0.57 & 16.03 & 0.70 & 8.8020 & 0.224 & 0.004 & 0.215 & 0.002 & 5.41 & 0.06 & $\mathrm{C}$ & \\
\hline J132433-624202(tm550016773) & 13:24:33.35 & $-62: 42: 02.40$ & 12.69 & 0.65 & 13.37 & 0.50 & 1.7601 & & & & & & & EA & $\mathrm{V}^{*} \mathrm{NX}$ Cen \\
\hline J132449-631304(tm5504k9109) & $13: 24: 49.24$ & $-63: 13: 04.60$ & 14.56 & 1.24 & 15.72 & 1.41 & 3.5099 & & & & & & & EA & \\
\hline J132457-633032(tm5506k8804) & $13: 24$ & $-63: 30: 32.00$ & 12.55 & 0.54 & 13.66 & 0.52 & 1.5775 & & & & & & & $\mathrm{EA}$ & \\
\hline J132500-631337(tm5504k10856) & $13: 25$ & $-63: 13: 37.21$ & 14.64 & 0.22 & 15.91 & 0.31 & & 0.405 & 0.036 & 0.151 & 0.008 & -0.06 & 0.03 & puls & \\
\hline J132531-633526(tm5506m1888) & $13: 25: 31.96$ & $-63: 35: 26.48$ & 14.96 & 1.36 & 16.15 & 1.47 & 1.6274 & & & & & & & EA & \\
\hline J132630-630945(tm5504m12359) & 13:26:30.11 & $-63: 09: 45.66$ & 10.18 & 0.52 & 11.77 & 0.64 & 12.8941 & 0.129 & 0.001 & 0.146 & 0.001 & 4.33 & 0.23 & $\mathrm{C}$ & $\mathrm{V}^{*}$ OO Cen \\
\hline $\mathrm{J} 132721-630110(\operatorname{tm} 550313554)$ & 13:27:21.68 & $-63: 01: 10.80$ & 10.98 & 0.80 & 12.67 & 0.97 & 15.2278 & 0.286 & 0.006 & 0.219 & 0.003 & 4.39 & 0.15 & $\mathrm{C}$ & $\mathrm{V}^{*}$ V881 Cen \\
\hline
\end{tabular}

Table 5. Catalogue of stars with $P>30$ days (sample).

\begin{tabular}{|c|c|c|c|c|c|c|c|c|c|}
\hline EROS2 GSA ID & RA(J2000) & $\overline{\mathrm{DEC}(\mathrm{J} 2000)}$ & $\left\langle R_{\mathrm{E}}\right\rangle$ & $\Delta R_{\mathrm{E}}$ & $\left\langle V_{\mathrm{E}}\right\rangle$ & $\overline{\Delta \Delta V_{\mathrm{E}}}$ & $P($ days $)$ & Type & Note \\
\hline $\mathrm{J} 132359-623229(\operatorname{tm} 550011488)$ & $13: 23: 59.86$ & $-62: 32: 29.63$ & 10.83 & 0.14 & 14.18 & 0.22 & 52.04 & SR & MSX5C G306.6758+00.0933 \\
\hline J132400-632057(tm550411003) & $13: 24: 00.60$ & $-63: 20: 57.82$ & 12.34 & 0.20 & 16.06 & 0.38 & 45.83 & $\mathrm{SR}$ & [CKS91] 13206-6305 \\
\hline J132419-632717(tm5506k3687) & $13: 24: 19.25$ & $-63: 27: 17.42$ & 11.56 & 0.34 & 14.94 & 5.43 & $>60 \mathrm{~d}$ & LPV & MSX5C G306.5942-00.8179 \\
\hline $\mathrm{J} 132427-631542(\operatorname{tm} 5504 \mathrm{k} 5591)$ & $13: 24: 27.10$ & $-63: 15: 42.08$ & 11.75 & 0.18 & 14.88 & 0.33 & $>60 \mathrm{~d}$ & LPV & [CKS91] 13211-6300 \\
\hline J132431-630539(tm5504k6408) & $13: 24: 31.78$ & $-63: 05: 39.51$ & 15.34 & 0.27 & 17.19 & 0.50 & 55.70 & & \\
\hline J132433-632201(tm5504m10330) & $13: 24: 33.05$ & $-63: 22: 01.64$ & 11.96 & 0.24 & 9.49 & 0.43 & 58.46 & & [CKS91] 13229-6252 \\
\hline $\mathrm{J} 132437-623119(\operatorname{tm} 5500 \mathrm{k} 7345)$ & $13: 24: 37.80$ & $-62: 31: 19.09$ & 12.4 & 0.21 & 15.94 & 0.47 & $>60 \mathrm{~d}$ & & \\
\hline J132440-633236(tm5506k6457) & $13: 24: 40.22$ & $-63: 32: 36.78$ & 11.67 & 0.34 & 15.46 & 0.42 & $>60 \mathrm{~d}$ & & MSX5C G306.6222-00.9099 \\
\hline J132444-632224(tm550417786) & $13: 24: 44.82$ & $-63: 22: 24.20$ & 10.79 & 0.44 & 14.13 & 1.19 & 55.00 & & IRAS 13214-6306; MSX5C G306.6524-00.7426 \\
\hline J161332-541002(gn4590k724) & $16: 13: 32.50$ & $-54: 10: 02.22$ & 13.14 & 0.84 & 16.49 & 1.79 & $>60 \mathrm{~d}$ & LPV & 2MASS 1613326-541004 \\
\hline J161332-541453(gn4590k673) & $16: 13: 32.45$ & $-54: 14: 53.91$ & 13.87 & 0.18 & 16.12 & 0.26 & 38.29 & SR & 2MASS 1613325-541454 \\
\hline
\end{tabular}




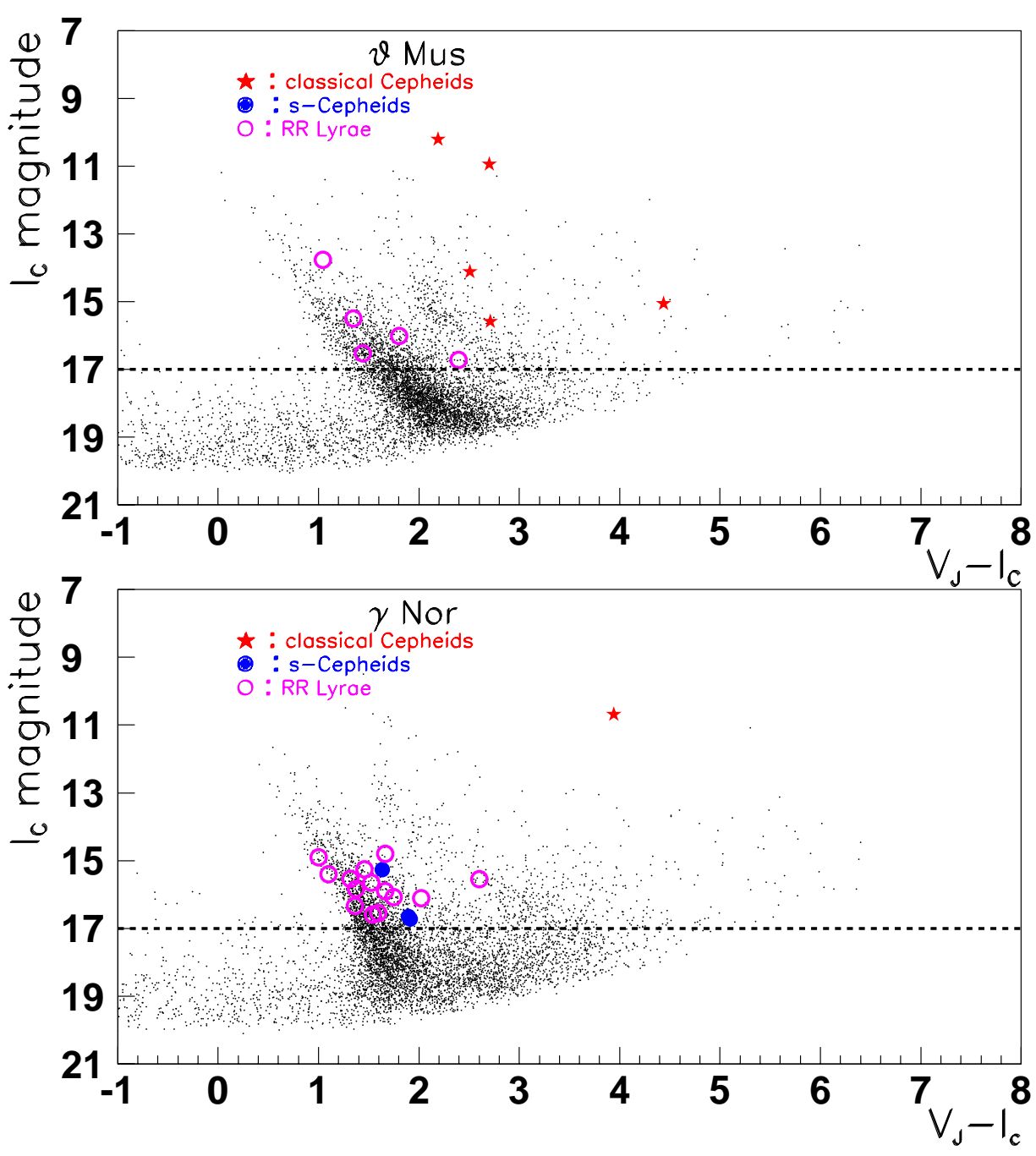

Fig. 9. Colour-magnitude diagrams $\left(I_{\mathrm{C}}\right.$ vs $\left.V_{\mathrm{J}}-I_{\mathrm{C}}\right)$ for the classical Cepheids (represented by stars $\left.\star\right)$, $s$-Cepheids (dots •), and RR Lyræ (open circles o).

Table 6. Period and coordinates obtained on OO Cen by SIMBAD, GCVS and this study.

\begin{tabular}{llll}
\hline \hline Source & SIMBAD & GCVS & This study \\
\hline object & $\mathrm{V}^{*}$ OO Cen & OO Cen & J132630-630945(tm5504m12359) \\
$P($ days $)$ & $\mathrm{n} / \mathrm{a}$ & 12.8805 & 12.894 \\
$\alpha(\mathrm{B} 1950)$ & $13: 23: 06.85$ & $13: 23: 09$ & $13: 23: 08.8$ \\
$\delta(\mathrm{B} 1950)$ & $-62: 54: 24.9$ & $-62: 54.0$ & $-62: 54: 11$ \\
\hline
\end{tabular}

observations. Therefore the comparison can only be carried out on 0.7 square degree. Furthermore, as pointed above, this comparison is restricted to stars with magnitude $I_{\mathrm{C}}<14$. A total of 118 EROS objects, 6 GCVS and $23 \mathrm{CKS} 91$ objects lie in this region. Three objects are common to the GCVS and CKS91 catalogues one. The overlap between the three catalogues represents only 19 objects. Among them one finds the most interesting ones, such as Cepheids OO CEN and V881 CEN (see Table 7). Some objects show a large difference between the two magnitude determinations. These are long period variables for which the mean magnitude is measured on only a part of the whole period, and thus ill determined in both surveys. Only 7 known variable stars are not recovered by our analysis (see Table 8). Conversely, 99 objects of our catalogue are not listed by CKS91. All of them are labelled Long Period Variable stars (LPV).

\section{Discussion}

The motivation of our search was to improve our knowledge of the distance distribution and of the extinction of the microlensing source stars used in Papers I and II. In the following we use the RR Lyræ which are well-known distance indicators and have been observed in all six directions that we investigated. The GSA fields having a high 
Table 7. Previously known variable stars recovered by this survey. For each star we give its name, the original catalogue, the type of variability, the period and the $I$ magnitude given (if any) in the catalogue and in this survey.

\begin{tabular}{|c|c|c|c|c|c|c|c|}
\hline Object & Catalogue & Type & $P($ days $)$ & $I$ & EROS ID & $P($ days $)$ & $I_{\mathrm{c}}$ \\
\hline OO Cen & GCVS & $\mathrm{C}$ & 12.8805 & 9.96 & $\begin{array}{c}\mathrm{J} 132630-630945 \\
(\operatorname{tm} 5504 \mathrm{~m} 12359)\end{array}$ & 12.8941 & 10.20 \\
\hline V881 Cen & GCVS & $\mathrm{C}$ & - & 10.57 & $\begin{array}{c}\mathrm{J} 132721-630110 \\
(\operatorname{tm} 550313554)\end{array}$ & 15.2278 & 10.97 \\
\hline V608 Cen & GCVS & $\mathrm{EB}$ & 1.6287 & 12.95 & $\begin{array}{l}\mathrm{J} 132948-630634 \\
(\operatorname{tm} 5505 \mathrm{~m} 13922)\end{array}$ & 1.7601 & 12.05 \\
\hline $\begin{array}{l}\text { CKS91 } \\
13246-6259\end{array}$ & CKS91 & EA & - & 13.29 & $\begin{array}{c}\mathrm{J} 132758-631449 \\
(\operatorname{tm} 550519598)\end{array}$ & 5.4918 & 13.03 \\
\hline $\begin{array}{l}\text { CKS91 } \\
13297-6244\end{array}$ & CKS91 & $\mathrm{E}$ & - & $\begin{array}{c}13.05 \\
1\end{array}$ & $\begin{array}{l}\text { J133311-630023 } \\
(\operatorname{tm} 5511 \mathrm{~m} 5801)\end{array}$ & 0.2141 & 13.20 \\
\hline $\begin{array}{l}\text { CKS91 } \\
13206-6305\end{array}$ & CKS91 & SR & - & 12.35 & $\begin{array}{c}\mathrm{J} 132400-632057 \\
(\operatorname{tm} 550411003)\end{array}$ & 45.83 & 12.34 \\
\hline $\begin{array}{l}\text { CKS91 } \\
13211-6300\end{array}$ & CKS91 & LPV & - & 11.73 & $\begin{array}{c}\mathrm{J} 132427-631542 \\
(\operatorname{tm} 5504 \mathrm{k} 5591)\end{array}$ & $P>60$ & 11.75 \\
\hline $\begin{array}{l}\text { CKS91 } \\
13214-6306\end{array}$ & CKS91 & SR & - & 10.51 & $\begin{array}{c}\text { J132444-632224 } \\
(\operatorname{tm} 550417786)\end{array}$ & 55.00 & 10.79 \\
\hline $\begin{array}{l}\text { CKS91 } \\
13214-6256\end{array}$ & CKS91 & SR & - & 12.85 & $\begin{array}{c}\mathrm{J} 132447-631201 \\
(\operatorname{tm} 5504 \mathrm{k} 8841)\end{array}$ & 52.39 & 12.77 \\
\hline $\begin{array}{l}\text { CKS91 } \\
13215-625\end{array}$ & CKS91 & LPV & - & 10.43 & $\begin{array}{c}\mathrm{J} 132454-631437 \\
(\operatorname{tm} 5504 \mathrm{k} 9938)\end{array}$ & $P>60$ & 10.23 \\
\hline $\begin{array}{l}\text { CKS91 } \\
13219-6301\end{array}$ & CKS91 & LPV & - & 11.81 & $\begin{array}{l}\text { J132519-631720 } \\
(\operatorname{tm} 5504113138)\end{array}$ & $P>60$ & 13.00 \\
\hline $\begin{array}{l}\text { CKS91 } \\
13229-6252\end{array}$ & CKS91 & LPV & - & 12.51 & $\begin{array}{c}\mathrm{J} 132433-632201 \\
(\operatorname{tm} 5504 \mathrm{~m} 10330)\end{array}$ & 58.46 & 11.96 \\
\hline $\begin{array}{l}\text { CKS91 } \\
13230-6251\end{array}$ & CKS91 & LPV & - & 12.48 & $\begin{array}{l}\mathrm{J} 132625-630645 \\
(\operatorname{tm} 5504 \mathrm{~m} 11603)\end{array}$ & $P>60$ & 12.41 \\
\hline $\begin{array}{l}\text { CKS91 } \\
13238-6227\end{array}$ & CKS91 & LPV & - & 12.63 & $\begin{array}{c}\mathrm{J} 132714-634338 \\
(\operatorname{tm} 550711058)\end{array}$ & $P>60$ & 10.78 \\
\hline $\begin{array}{l}\text { CKS91 } \\
13238-6254\end{array}$ & CKS91 & LPV & - & 12.21 & $\begin{array}{c}\mathrm{J} 132719-633644 \\
(\operatorname{tm} 550711752)\end{array}$ & $P>60$ & 11.91 \\
\hline $\begin{array}{l}\text { CKS91 } \\
13246-6306\end{array}$ & CKS91 & M & - & 12.46 & $\begin{array}{l}\mathrm{J} 132803-632200 \\
(\operatorname{tm} 5505110106)\end{array}$ & $P>60$ & 13.99 \\
\hline $\begin{array}{l}\text { CKS91 } \\
13247-6244\end{array}$ & CKS91 & $\mathrm{SR}$ & - & 12.66 & $\begin{array}{c}\mathrm{J} 132813-630013 \\
(\operatorname{tm} 5510 \mathrm{k} 2095)\end{array}$ & 44.80 & 12.72 \\
\hline $\begin{array}{l}\text { CKS91 } \\
13264-6256\end{array}$ & CKS91 & LPV & - & 12.37 & $\begin{array}{r}\text { J132948-631227 } \\
(\operatorname{tm} 5510 n 4312)\end{array}$ & $P>60$ & 12.56 \\
\hline $\begin{array}{l}\text { CKS91 } \\
13289-6308\end{array}$ & CKS91 & M & - & 12.65 & $\begin{array}{l}\text { J133222-632339 } \\
(\operatorname{tm} 5513 \mathrm{k} 12129)\end{array}$ & $P>60$ & 12.65 \\
\hline
\end{tabular}

non-uniform absorption, we give only an average reddening towards our fields, based on EROS data alone.

For each selected RR Lyræ we estimate the extinction $A(V)=2.4 \times E(V-I)$ using the standard extinction coefficients (Schlegel et al. 1998; Stanek 1996). The colour excess $E(V-I)$ is derived from the colour that we measured and their intrinsic colour: $(V-I)_{0}^{\mathrm{RRab}}=$ 0.4 and $(V-I)_{0}^{\mathrm{RRc}}=0.2$ (Alcock et al. 1998). The uncertainty on individual extinctions is estimated to be $0.5^{\mathrm{mag}}$. This error includes the $0.2^{\mathrm{mag}}$ uncertainty on the EROS colour measurement (see Eq. (1)) and a $0.1^{\text {mag }}$ magnitude uncertainty on the intrinsic colour. The absolute magnitude of RR Lyræ is $M_{V}^{\mathrm{RR}} \sim 0.71$ with a precision of $0.07^{\mathrm{mag}}$ (Gould \& Popowski 1998). Finally, we estimate the distance to each star simply by using the relation $m-M=5 \times(\log (d)-1)+A$. The typical uncertainty on the distance is $20 \%$. The left panels of Fig. 11 show the obtained extinction $A(V)$ versus the calculated distance. The mean extinction of the RR Lyræ is $A(V) \sim 3.6 \pm 0.2 \mathrm{mag}$ towards $\theta$ Mus and $3.3 \pm 0.1$ towards $\gamma$ Nor, with a 


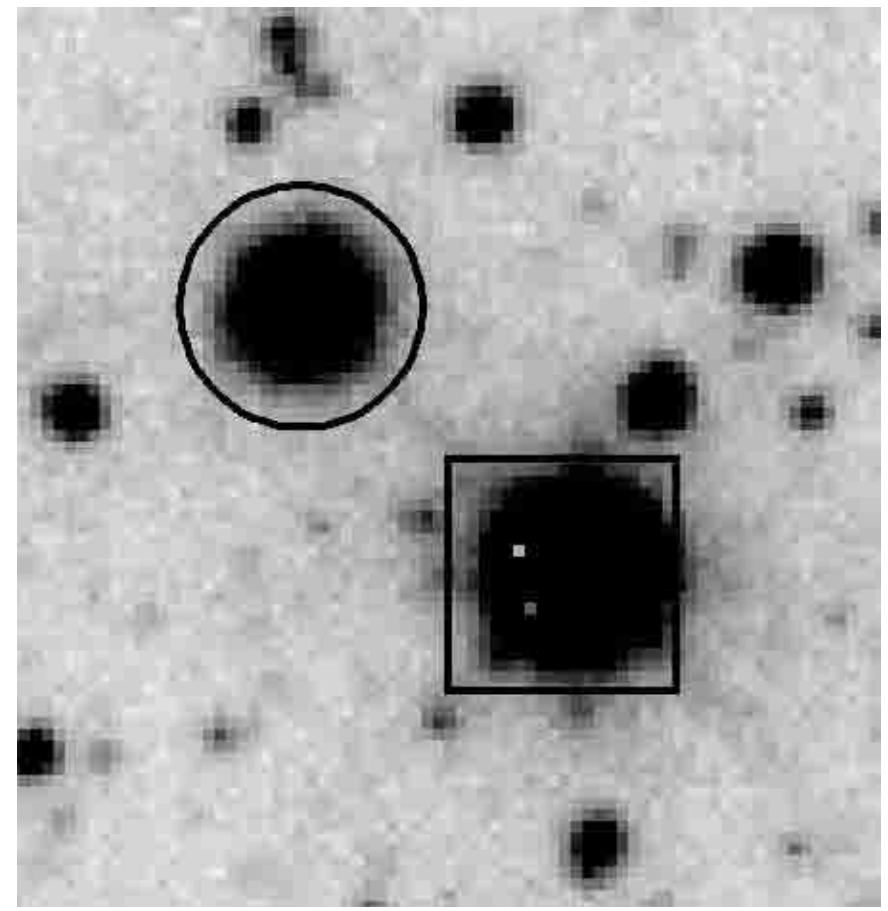

Fig. 10. Finding chart of the star OO Cen - J132630630945(tm5504m12359). The position given by the SIMBAD database is shown by the square which is $10^{\prime \prime}$ wide, while the EROS position is shown by the circle.

Table 8. Previously known variable stars not recovered by this survey. For each of them we give (where possible) their type of variability, their period and the reason for their absence in the catalogue.

\begin{tabular}{lcccl}
\hline \hline Object & Type & $P$ (days) & catalogue & comment \\
\hline HQ Nor & EB & 90.9 & GCVS & too long period \\
HY Nor & Mira & 236 & GCVS & $\begin{array}{l}\text { close to } \\
\text { the CCDs gap }\end{array}$ \\
& & & GCVS & fails cut 6 \\
UW Nor & EA & 8.4860 & fails cut 6 \\
$13214-6256$ & LPV & & CKS91 & fails cut 6 \\
$13218-6254$ & LPV & & CKS91 & fails cut 6 \\
$13248-6249$ & LPV & & CKS91 & fails cut 6 \\
$13232-6249$ & LPV & & CKS91 & fails cut 6 \\
\hline
\end{tabular}

dispersion of 1 mag. The mean distance of the RR Lyræ is $\sim 4.7 \pm 0.3 \mathrm{kpc}$ towards $\theta$ Mus and $5.3 \pm 0.2 \mathrm{kpc}$ towards $\gamma$ Nor. The dispersion of the values is $1.4 \mathrm{kpc}$ which reflects the spread in distance of disc stars. The disc population contributes to the sources of the microlensing events that we observe in the Galactic plane. A model based on the distribution of matter in the disc and the luminosity function of neighbooring stars has been used in Derue (1999) to estimate the distance of the star population from the disc. The distance of RR Lyræ is in good agreement with the one obtained with this model.
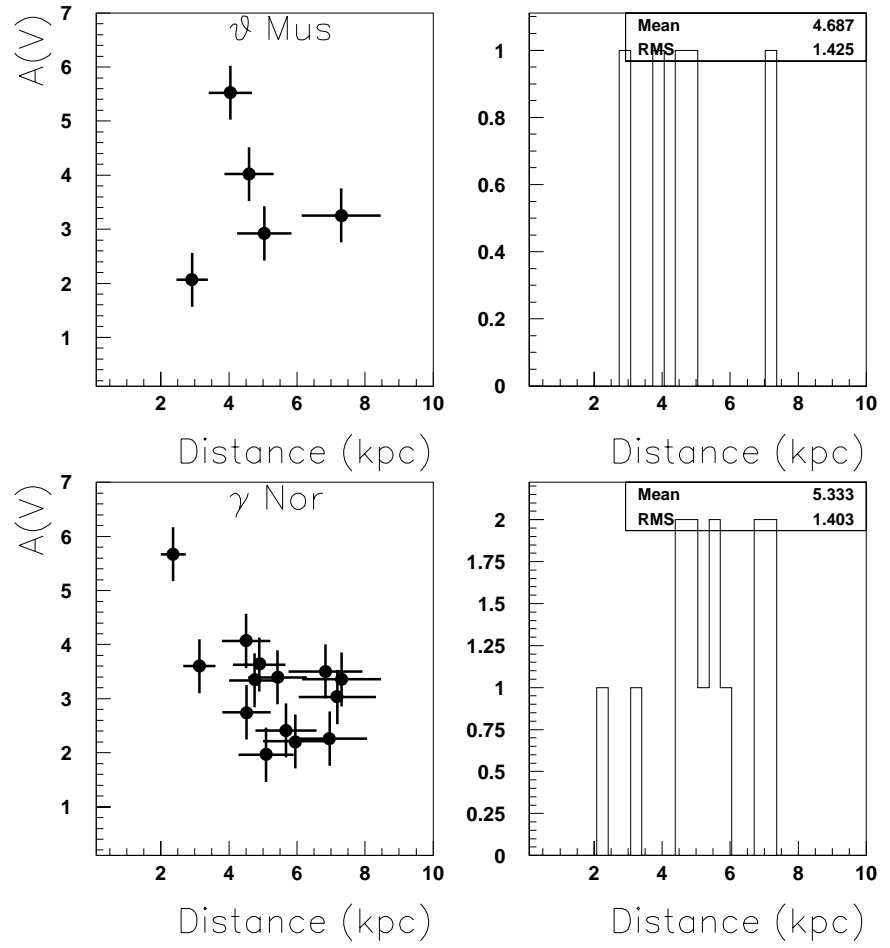

Fig. 11. Extinction $A(V)$ versus the distance of RR Lyræ (in kpc) towards $\theta$ Mus and $\gamma$ Nor. The right panels show the inferred distance distribution.

\section{Conclusion}

In the course of our program dedicated to microlensing events, we have devoted a fraction of observing time to the search for variable stars in six directions of the Galactic plane. This exploratory campaign, that lasted three months, led to the discovery of 1362 variable stars. Among them we identified 9 Cepheids, 19 RR Lyræ, 34 Miras, 176 eclipsing binaries and 266 Semi-Regular variable stars. We have set up a catalogue of all of the 1362 stars and cross-identified it with several other catalogues. In particular a comparison with the GCVS and the CKS91 catalogues shows that only a small fraction $(\sim 15 \%)$ of the objects that we have identified appear in those two. Among the stars most appropriate to be used as distance indicators, the Cepheids turned out to be too few to warrant a particular study. As far as RR Lyræ are concerned, we have determined their mean distance and found it to be $\sim 5 \mathrm{kpc}$. Yet the statistics being quite limited, we are considering pursuing this effort by launching a longer search for variable stars based on the results of this first campaign.

Acknowledgements. The WCSTools package was made available to us thanks to the work of Doug Mink, NASA/GSFC, Harvard. The Skycat/Gaia tool is the result of a joint effort by the computer staff of ESO Garching and from the Starlink Project, UK. 


\section{References}

Afonso, C., Albert, J. N, Amadon, A., et al. (EROS coll.) 1999, CEA Saclay Report Dapnia-SPP 99/25. See also http://www-dapnia.cea.fr/Spp/Experiences/EROS/ Cepheides/catalog_cep.html

Alcock, C., Allsman, R. A., \& Alves, D. R. (MACHO coll.) 1998, ApJ, 492, 190

Ansari, R. 1996, Vistas in Astron., 40, 519

Antonello, E., \& Poretti, E. 1986, A\&A, 169, 149

Beaulieu, J.-P., Grison, P., Tobin, W., et al. (EROS coll.) 1995, ApJ, 303, 137

Beaulieu, J.-P., \& Sasselov, D. 1996, Variable Stars and the Astrophysical Returns of Microlensing Surveys, ed. R. Ferlet, J. P. Maillard, \& B. Raban, 193

Beichman, C. A., Neugebauer, G. A., Habing, H. J., Clegg, P. E., \& Chester, T. J. 1988, Explanatory Supplement to the IRAS Catalogs and Atlases

Caldwell, J., Keane, M., \& Schechter, P. 1991, AJ, 101, 1763

Cioni, M.-R. L., Marquette, J.-B., Loup, C., et al. 2001, A\&A, 377,945

Derue, F. 1999, Ph.D. Thesis, CNRS/IN2P3, LAL report 9914, Université Paris XI Orsay, also available at the URL http://www.lal.in2p3.fr/presentation/bibliotheque/ publications/Theses99.html

Derue, F., Afonso, C., Alard, C., et al. (EROS coll.) 1999, A\&A, 351, 87

Derue, F., Afonso, C., Alard, C., et al. (EROS coll.) 2001, A\&A, 373, 126

Egan, M. P., Price, S. D., Moshir, M. M., et al. 1999, AFRLVS-TR-1999-1522

Fouqué, P., Chevallier, L., Cohen, M., et al. 2000, A\&AS, 141, 313

Georgelin, Y. M, Amram, P., Georgelin, Y. P., et al. 1994, A\&AS, 108, 513

Gould, A., \& Popowski, P. 1998, ApJ, 508, 844
Grison, P. 1994, A\&A, 289, 404

Grison, P., Beaulieu, J.-P., Pritchard, J.-D., et al. (EROS coll.) 1995, A\&AS, 109, 447

Harris, J., \& Zaritsky, D. 1999, AJ, 117, 2831

Kholopov, P. N. 1985, in General Catalogue of Variable Stars, vol. 1 (Moscow Nauka Pub. House)

Madore, B. F., \& Freedman, W. L. 1991, PASP, 103, 933

Mansoux, B. 1997, Ph.D. Thesis, CNRS/IN2P3, LAL report 97-19, Université Paris 7

Mink, D. G. 1999, Astronomical Data Analysis Software and Systems VIII, ASP Conf. Ser., ed. D. Mehringer, R. Plante, \& D. Roberts, 498

Paczyński, B. 1996, invited talk at the Extragalactic Distance Scale STScI Symp., May 7-10, 1996, Baltimore, Maryland, USA [astro-ph/9608094]

Palanque-Delabrouille, N., Afonso, C., Albert, J.-N., et al. (EROS coll.) 1998, A\&A, 332, 1

Regnault, N. 2000, Ph.D. Thesis, Université Paris VII, LALCNRS/IN2P3 Report 00/65. also available at the URL http://www.lal.in2p3.fr/presentation/bibliotheque/ publications/Theses00.html

Russeil, D., Amram, P., Georgelin, Y. P., et al. 1998, A\&AS, 130, 119

Scargle, J. D. 1982, ApJ, 263, 835

Schwarzenberg-Czerny, A. 1996, ApJ, 460, L107

Schlegel, D. J., Finkbeiner, D. P., \& Davies, M. 1998, AJ, 500, 525

Skrutskie, M. F., Schneider, S. E., Stiening, R., et al. (2MASS Coll) 1997, Proc. Workshop The Impact of Large Scale Near-IR Sky Surveys, 25

Stanek, K. Z. 1996, ApJ, 460, L37

Udalski, A., Szymański, M., Kubiak, M., et al. (OGLE Coll.) 1998, Act. Astr., 48, 1

Udalski, A., Soszynski, I., Szymański, M., et al. (OGLE Coll.) 1999, Act. Astr., 49, 45 